\title{
Construction of an anthropopathic abdominal phantom for accuracy validation of deformable image registration
}

\author{
Y.L. Liao, H.B. Chen, L.H. Zhou* and X. Zhen* \\ Department of Biomedical Engineering, Southern Medical University, Guangzhou, Guangdong, China
}

\begin{abstract}
The purpose of this study is to design and fabricate an anthropopathic abdominal phantom for accuracy evaluation of deformable image registration (DIR) algorithms in adaptive radiation therapy. The constructed deformable organs, including the liver, kidney, spleen and stomach, are made of mixture of polyvinyl chloride (PVC) and softener dioctyl terephthalate, while the rigid structures, i.e. vertebrae, are made of white cement. Relation between the PVC-softener blending ratio and organ CT number is studied, and three-dimensional printing technic is employed to create highly anthropopathic organs in terms of organ shape and density. Detailed steps for phantom construction, landmark point placement and choice of phantom ingredients and construction recipe are introduced. Preliminary results of the mechanical properties of the fabricated organs are also presented. The experimental results indicate that the constructed phantom has satisfactory elastic characteristics and close CT number with corporal organs, and can potentially be applied to simulate real abdominal organ deformation in geometric accuracy validation of DIR algorithms.
\end{abstract}

Keywords: Three-dimensional deformable phantom, deformable image registration, PVC, phantom construction

\section{Introduction}

Deformation image registration (DIR) is one of the key technics in adaptive radiation therapy (ART). It is mainly used for 1) auto-contouring of the target and organs at risk (OARs) to facilitate the process of treatment re-planning and 2) dose mapping and accumulation by employing the resulting deformation vector fields (DVFs) generated from DIR. The DIR accuracy is crucial in ART since it is closely related to the accuracy of treatment re-planning and dose accumulation. Physical deformable phantom is hitherto one of the most reliable means for DIR accuracy validation since it can measure the geometric accuracy by calculating residual errors of phantom implanted landmarks. Specifically, DIR is performed on the scanned CT images before and after deformation, and the resulting deformation vector filed can be applied to deform the implanted landmarks inside the phantom and to measure the residual errors by comparing the calculated and the ground truth landmarks offsets. A stable and reusable deformable phantom is therefore necessary for the purpose of accurate measurement of DIR performance.

Many efforts have been made in the design and construction of deformable phantom in last decades. For example, Kirby et al. [1] proposed a two-dimensional deformable head phantom, in which several implanted landmarks can be tracked and measured via optical image. Graves et al. [2] presented

\footnotetext{
${ }^{*}$ Corresponding authors: L.H. Zhou and X. Zhen, Department of Biomedical Engineering, Southern Medical University, Guangzhou, Guangdong 510515, China. E-mail: smart@smu.edu.cn (LZ), xinzhen@ smu.edu.cn (XZ).
}

0928-7329/16/\$35.00 @ 2016 - IOS Press and the authors. All rights reserved

This article is published online with Open Access and distributed under the terms of the Creative Commons Attribution NonCommercial License. 
a deformable head and neck phantom embedded with dosimeters and realistic organs that constructed from contours of CT images for ART quality control. However, the two aforementioned phantoms are two-dimensional ones, which can not simulate the complex three-dimensional deformation in reality. Some researchers focus on more comprehensive three-dimensional deformable phantoms. For instance, Singhrao et al. [3] reported a three-dimensional head-and-neck phantom for validation of multimodality image registration in ART. Kashani et al. [4,5] designed a simple and reusable abdominal deformable phantom, incorporating with respiratory movement simulation. Chang et al. [6] constructed a deformable lung phantom with motor control to simulate respiratory movement. Hungr et al. [7] proposed a prostate phantom embedded with anthropopathic structures which can be applied to ultrasound, CT and MR imaging. However, most of these phantoms are constructed based on simplified organ models, which might oversimplify the real organ deformation and will bring potential errors to the validation process [8].

In this paper, a deformable abdominal phantom is fabricated for the potential use of registration accuracy evaluation of DIR algorithms. Detailed steps of phantom construction, landmark point placement and choice of phantom ingredients and construction recipe are introduced. The developed organs inside the phantom are described and preliminary results of the mechanical properties of the phantom are also presented.

\section{Material and method}

The organs, including the liver, kidney, spleen, stomach and vertebra, inside the abdominal phantom are developed based on contours from the CT images of a clinical female patient. The organs on the CT images are firstly delineated manually by an experienced physician. Then, the marching cubes algorithm [9] is used to transfer the segmented masks to the surface meshes, which is to build the moulds of the organs by three-dimensional printing. All of the printed moulds are negative of the organs with a wall thickness of $2 \mathrm{~mm}$.

The polyvinyl chloride (PVC) is employed to fabricate the deformable tissues since it can provide ideal mechanical and imaging characteristics, and it has a sufficiently large range of elastic modulus and is inexpensive and simple to manufacture [7]. In order to simulate different organ elasticity and CT numbers, gel made of different mixtures of PVC and the softener dioctyl terephthalate is used as the deformable material in the phantom. CT scans are performed on sixteen PVC samples with increasing blending ratio, and a polynomial fit is then obtained to describe the relation between the CT number and the PVC-softener blending ratio.

Construction of the deformable phantom includes a laboratory hot plate, a glass rod, a balance and a $1000 \mathrm{ml}$ beaker (Figs 1a and 1b). The mixture of PVC and softener is heated in the beaker at a hot plate temperature of $280^{\circ} \mathrm{C}$ for $30 \mathrm{~min}$ until polymerized (Fig. 1b). During the heating, continuously stirring with a glass rod is necessary to avoid air bubbles in the mixture. In addition to stirring, it is helpful to preheat the moulds to a certain temperature to prevent the PVC from curing too quickly around the thin mould walls, thus trapping the escaping air bubbles. The solution is poured into the printed organ moulds and cooled at room temperature (Fig. 1d). In order to facilitate mould unloading, a thin layer of lubricant, e.g. Vaseline, is applied to the inner wall of the moulds (Fig. 1e).

Metal bearing balls (BBs) with diameter of $1 \mathrm{~mm}$ are embedded inside each gel phantom as fiducial landmarks for DIR accuracy evaluation (Fig. 1f). This is done by pouring the PVC solution into the mould in layers, letting each layer to cool at its surface to be firm enough to hold the BBs without them sinking, and then cover them with another layer of PVC (Fig. 1c). 

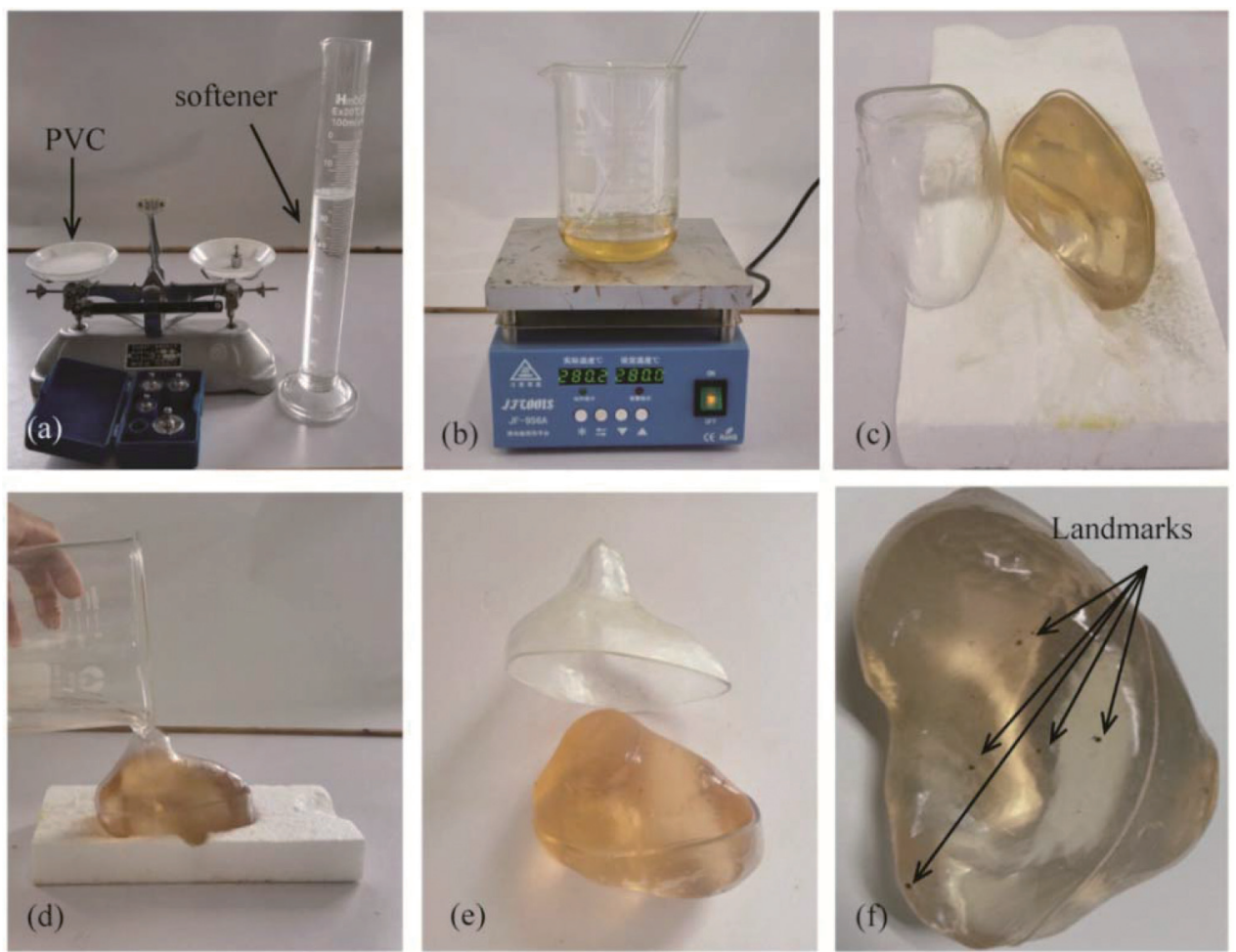

Fig. 1. Construction steps of the deformable phantom. (a) Measuring materials. (b) Heating the PVC mixture. (c) Placing the landmarks on the first layer of PVC after it is cooled. (d) Pouring the remainder molten PVC into the mould. (e) Unloading organ from the mould after curing. (f) The constructed organ (arrows indicate the implanted landmarks).

For rigid bone structures, including $1 \sim 3$ lumbar vertebrae and the $8 \sim 12$ thoracic vertebrae, are also constructed by moulding. White cement is used as the bone material since it has satisfactory rigidity and similar CT number with real bone $(\sim 1000 \mathrm{HU})$. The liquid white cement is poured into each bone moulds and allow it to solidify overnight.

\section{Results}

\subsection{Organs construction}

The constructed organs are shown in Fig. 2. All the organs in the phantom are based on the contours delineated manually in a CT image by an experienced physician (Fig. 2a). Figs 2bíC2g exhibits the constructed organs in the phantom, including the right and left kidney (Figs $2 b$ and $2 c$ ), the spleen (Fig. 2d), the stomach (Fig. 2e) and the left and right lobe of liver (Figs $2 \mathrm{f}$ and 2g), with organ sizes of $92 \mathrm{~mm} \times 57 \mathrm{~mm} \times 45 \mathrm{~mm}$ (b), $97 \mathrm{~mm} \times 55 \mathrm{~mm} \times 42 \mathrm{~mm}$ (c), $114 \mathrm{~mm} \times 82 \mathrm{~mm} \times 71 \mathrm{~mm}$ (d), $127 \mathrm{~mm}$ $\times 65 \mathrm{~mm} \times 34 \mathrm{~mm}(\mathrm{e}), 94 \mathrm{~mm} \times 122 \mathrm{~mm} \times 58 \mathrm{~mm}(\mathrm{f}), 159 \mathrm{~mm} \times 157 \mathrm{~mm} \times 115 \mathrm{~mm}(\mathrm{~g})$, respectively. All these organs are with 1:1 scale to their real sizes. The top left subfigures in Figs $2 \mathrm{~b}-2 \mathrm{~g}$ represent the surface meshes generated through the marching cubes algorithm, and the lower left subfigures show the three-dimensional printed moulds according to the surface meshes with extrusive entry for PVC pouring (arrows in Figs $2 \mathrm{~b}-2 \mathrm{~g}$ ). The final constructed organs are shown in the right subfigures in Figs $2 \mathrm{~b}-2 \mathrm{~g}$. 


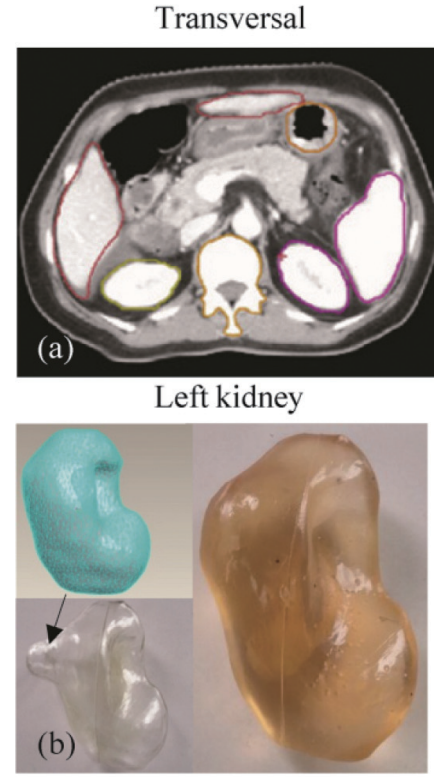

Stomach

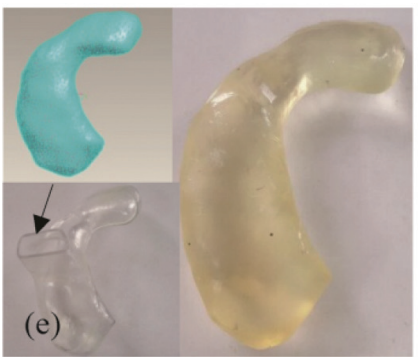

Coronal

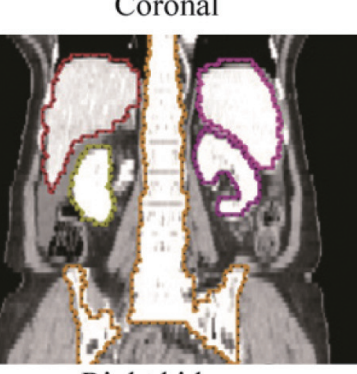

Right kidney

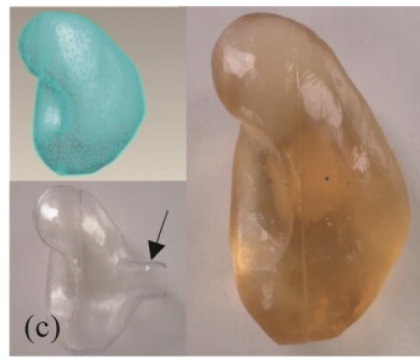

Liver(left lobe)

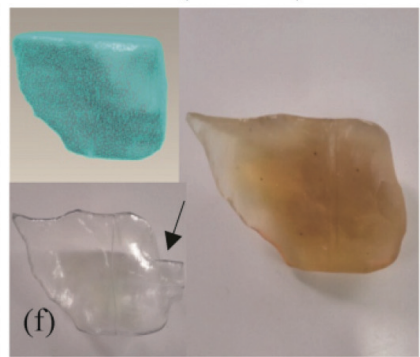

Sagittal

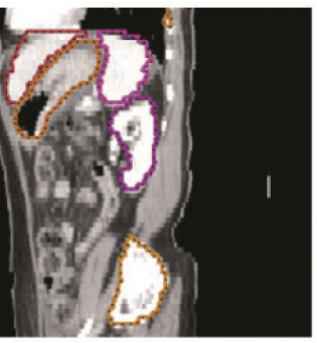

Spleen

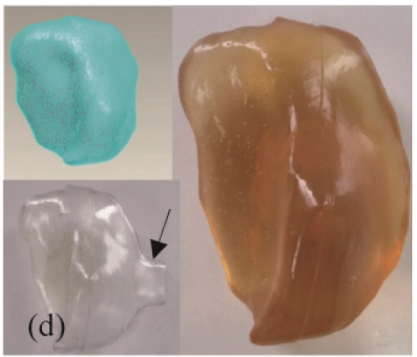

Liver(right lobe)

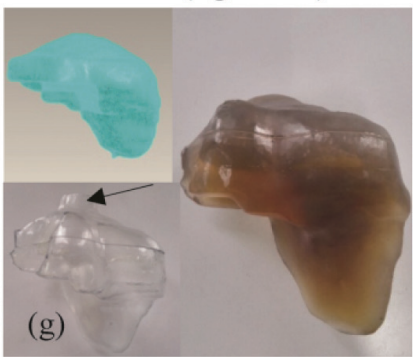

Fig. 2. Constructed organs. (a) Delineated abdominal organs. (b)-(g) The top left subfigures represent the surface meshes generated through the marching cubes algorithm. The lower left subfigures show the three-dimensional printed moulds. The right subfigures are the constructed organs. Arrows indicate the extrusive entry for PVC pouring.

\subsection{Vertebra construction}

All the vertebras are firstly three-dimensionally printed using Acrylonitrile Butadiene Styrene (ABS) according to their delineated contours, and the negative moulds for each organ are then created using the PVC polymer. The negative moulds are separated into two parts in order to facilitate the mould unloading. Liquid white cement is poured into the moulds, and it takes about two days to solidify. Figure 3 shows the constructed vertebras.

\subsection{CT number of constructed organs}

To find out the relationship between the CT number and the blending ratio of PVC powder and softener, sixteen PVC polymer samples are made with the blending ratio ranging from $8 \times 10^{-2} \mathrm{~g} / \mathrm{mL}$ to $23 \times 10^{-2} \mathrm{~g} / \mathrm{mL}$ with interval of $1 \times 10^{-2} \mathrm{~g} / \mathrm{mL}$, and CT scans are then performed to obtain the mean CT numbers of each sample. As shown in Fig. 4, evident linear relationship is found between the blending ratio and the CT numbers, and a linear fit can be obtained as $\mathrm{y}=6.9083 \mathrm{x}-65.5645$, where $\mathrm{x}$ and 

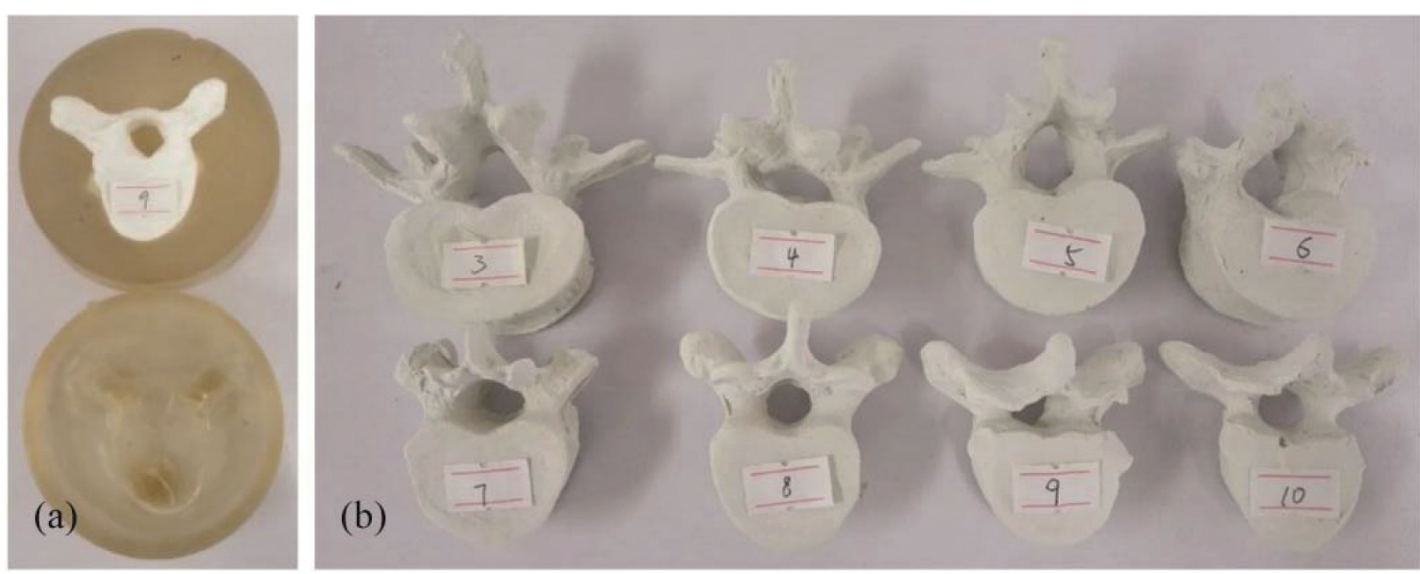

Fig. 3. (a) Negative mould of the vertebra. (b) Constructed vertebras.

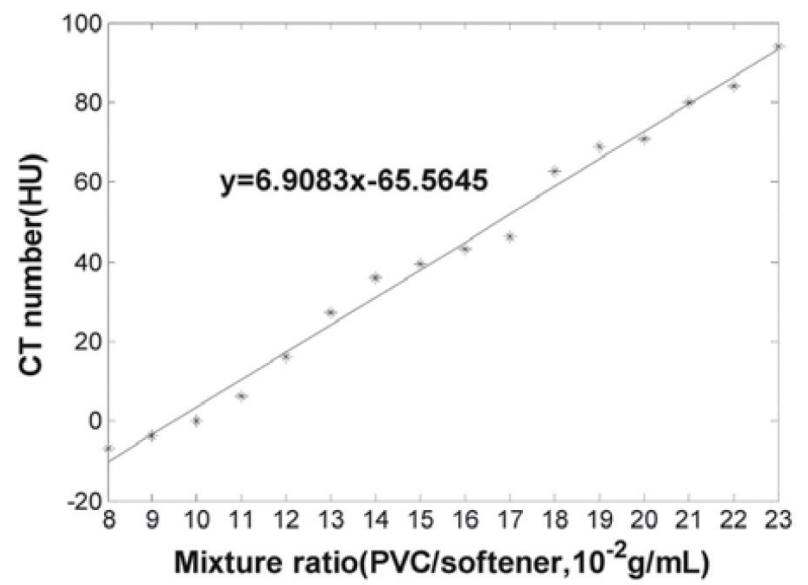

Fig. 4. The relationship between the blending ratio $\left(10^{-2} \mathrm{~g} / \mathrm{mL}\right)$ of PVC and softener and the CT number (HU).

y represent the blending ratio and CT number, respectively. The CT number of the samples range from -10 to 100 , which cover most of the abdominal soft tissues and organs. Based on the fitted relationship, blending ratio of our target organs can be computed. Table 1 shows the amount and blending ratio of the PVC and softener, as well as the target CT numbers and the finally obtained mean CT number for each organ. It is observed that the obtained mean CT numbers of the organs are close to the target ones, and the deviations are in a reasonable range.

\subsection{Elasticity of constructed organs}

To seek out the relationship between the organ elasticity and CT number, the compression stress-strain for sixteen PVC polymer samples are measured using a mechanical characterization machine (BOSE, 3220-ATSeries II, USA, as shown in Fig. 5a). The resulting displacement-force curves are shown in Fig. 5b. For all the samples, the curve shows viscoelastic behavior, with an increase in elastic modulus with increasing strain. Elastic modulus is proportional to the density of the materials, indicated by increased force is needed for higher density samples to undertake same degree of deformation. 
Table 1

The amount and blending ratio of materials, as well as the target and obtained mean CT numbers for all the constructed organs

\begin{tabular}{lccccc}
\hline Organ & $\begin{array}{c}\text { PVC } \\
(\mathrm{g})\end{array}$ & $\begin{array}{c}\text { Softener } \\
(\mathrm{mL})\end{array}$ & $\begin{array}{c}\text { Blending ratio } \\
\left(10^{-2} \mathrm{~g} / \mathrm{mL}\right)\end{array}$ & $\begin{array}{c}\text { Target CT } \\
\text { number }(\mathrm{HU})\end{array}$ & $\begin{array}{c}\text { Obtained mean } \\
\text { CT number }(\mathrm{HU})\end{array}$ \\
\hline Stomach & 23.1 & 150 & 15.4 & 41 & $45.0( \pm 5.0)$ \\
Kidney & 31.4 & 200 & 15.7 & 43 & $46.5( \pm 7.2)$ \\
Spleen & 51.9 & 300 & 17.3 & 54 & $57.8( \pm 6.4)$ \\
Liver & 74.4 & 400 & 18.6 & 63 & $69.1( \pm 7.1)$ \\
\hline
\end{tabular}
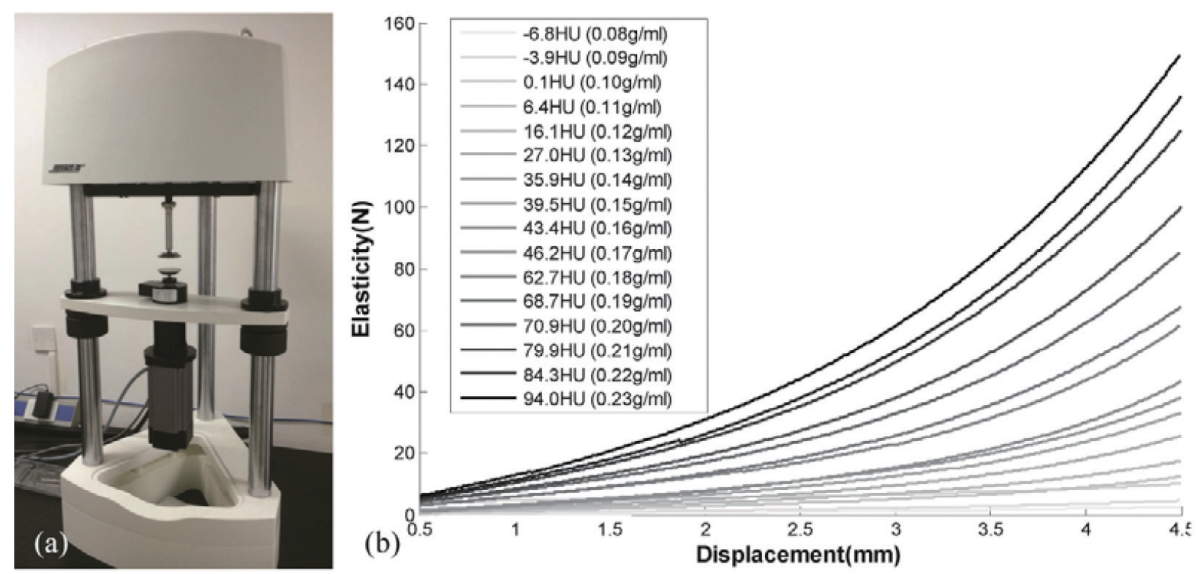

Fig. 5. (a) Mechanical characterization machine used to measure the compression stress of the PVC polymer samples. (b) Displacement-force curves of sixteen samples with different blending ratios and CT numbers.

\section{Discussion and conclusion}

In this study, we design and construct an anthropopathic deformable abdominal phantom for the purpose of registration accuracy validation of DIR algorithms. The material of the phantom is easily obtainable, low price, nontoxic and environmental friendly. There are several merits of the constructed phantom for the potential use of DIR accuracy validation: Firstly, all the organs are made via three-dimensional printing technic, based on the corresponding contours on the CT image from a clinical cancer patient, thus, it has highly anthropopathic anatomical shapes with the realistic organs. Secondly, the deformable organs are made of the different mixture of PVC and softener dioctyl terephthalate, resulting satisfactory elastic property and close CT numbers with the corporal organs. Thirdly, fiducial landmarks have been implanted inside each organ that can be used to quantitatively assess the DIR accuracy organ-wise. Finally, this phantom is recyclable and can be used routinely for DIR accuracy evaluation in ART.

To note that this work is an ongoing project and more sophisticated functions, such as simulating respiratory movement, construction of hollow structures (e.g. the rectum and bladder), and embedding dosimeters for absorb dose measurement, etc. [10,11], need to investigate before the phantom is assembled for final clinical use. We would like to include these works into our future studies.

\section{Acknowledgments}

This work is supported in part by the National Natural Science Foundation of China (no 81428019 and no 81301940), the Guangdong Natural Science Foundation (2015A030313302) and the 2015 Pearl River S\&T Nova Program of Guangzhou (201506010096). 


\section{References}

[1] N. Kirby, C. Chuang and J. Pouliot, A two-dimensional deformable phantom for quantitatively verifying deformation algorithms. Med Phys 38, 4583 (2011).

[2] Y.J. Graves, A. Smith, D. Mcilvena, Z. Manilay, Y.K. Lai, R. Rice, L. Mell, X. Jia, S.B. Jiang and L. Cerviño, A deformable head and neck phantom with in-vivo dosimetry for adaptive radiotherapy quality assurance. Med Phys 42, 1490 (2015).

[3] K. Singhrao, N. Kirby and J. Pouliot, A three-dimensional head-and-neck phantom for validation of multimodality deformable image registration for adaptive radiotherapy. Med Phys 41, 121709 (2014).

[4] R. Kashani, M. Hub, M.L. Kessler and J.M. Balter, Technical note: A physical phantom for assessment of accuracy of deformable alignment algorithms. Med Phys 34, 2785 (2007).

[5] R. Kashani, K. Lam, D. Litzenberg and J. Balter, Technical note: A deformable phantom for dynamic modeling in radiation therapy. Med Phys 34, 199 (2007).

[6] J. Chang, T.S. Suh and D.S. Lee, Development of a deformable lung phantom for the evaluation of deformable registration. J Appl Clin Med Phys 11, 3081 (2010).

[7] N. Hungr, J. Long, V. Beix and J. Troccaz, A realistic deformable prostate phantom for multimodal imaging and needleinsertion procedures. Med Phys 39, 2031 (2012).

[8] M. Serban, E. Heath, G. Stroian, D.L. Collins and J. Seuntjens, A deformable phantom for 4D radiotherapy verification: Design and image registration evaluation. Med Phys 35, 1094 (2008).

[9] W.E. Lorensen and H.E. Cline, Marching cubes: A high resolution 3D surface construction algorithm. SIGGRAPH Comput. Graph. 21, 163 (1987).

[10] U.J. Yeo, M.L. Taylor, L. Dunn, T. Kron, R.L. Smith and R.D. Franich, A novel methodology for 3D deformable dosimetry. Med Phys 39, 2203 (2012).

[11] C.J. Niu, W.D. Foltz, M. Velec, J.L. Moseley, A. Al-Mayah and K.K. Brock, A novel technique to enable experimental validation of deformable dose accumulation. Med Phys 39, 765 (2012). 